\title{
Therapeutic application of monoclonal antibodies: a review
}

\author{
Haben FESSEHA ${ }^{1 \otimes}$, Tadesse DEGU ${ }^{2}$ and Dereje ENDASHAW ${ }^{2}$ \\ ${ }^{1}$ School of Veterinary Medicine, Wolaita Sodo University, P. O. Box 138, Wolaita Sodo, Ethiopia \\ ${ }^{2}$ College of Veterinary Science, Mekelle University, Mekelle, Ethiopia \\ Corresponding author's Email: tseyon.h@gmail.com; (D) ORCiD: 00oo-0001-6516-3036
}

\section{ABSTRACT}

Introduction. Antibodies are an important class of proteins produced when a foreign entity elicits an immune response in the body. There are 5 major classes of antibodies, IgM, IgG, IgA, IgD, and IgE. The structure and immune function of immunoglobulins differ. An antibody contains two light and two heavy chains, which are linked by multiple disulphide bonds. Variable regions are found on light and heavy chains, known as the fragment antigen-binding (Fab) region, and a constant region, which is also known as the fragment crystallizable (Fc) region. Antibodies are, as a class, broad-spectrum antimicrobial agents with activity against all classes of pathogens. However, individual antibodies are usually pathogen-specific. Monoclonal antibodies, which specifically recognize one epitope of the cognate antigen, can be generated by using antibody engineering techniques such as hybridoma, phage display, and transgenic technologies. Removal of the entire constant region or part of the whole Fc portion generates antibody fragments such as Fab, scFv, and diabodies. Better tissue or tumor penetration characteristic of antibody fragments make them suited for the therapy overusing the whole antibody, hence most suited for therapy. Aim. Nowadays, it is possible to use antibodies for different therapeutic applications by

\section{Review Article}

PII: S225199392000008-10

Rec. Ol July 2020

Rev. 20 September 2020

Pub. 25 September 2020

\section{Keywords}

Antibody engineering, Monoclonal antibodies, Therapeutic antibodies modifying either their structural or functional properties.

\section{INTRODUCTION}

The introduction of a trail to treat infectious human diseases using animal antibodies dates back a century [1]. In the 1930 s and 1940 s antibiotics such as sulfonamides and penicillin replaced serum therapy. The decline of therapeutic horse and rabbit antisera is caused by the associated toxicity of serum sickness [2]. Polyclonal immunoglobulin and human antisera preparation were better tolerated; however, lot-to-lot variation, low content of specific antibodies and infectious agents such as HIV and hepatitis viruses are the limitations [3].

In the late 1970s and early 1980s, the discovery and diffusion of monoclonal antibody (MAb) technology become a new promising area of human therapeutics. MAbs could be selected with exquisite specificity. They function on various components of the immune system such as antibody-dependent cell cytotoxicity (ADCC) and complement, and they showed a high biological half-life in blood and tissues, rendering them effective for prophylactic use. Due to their biological nature, the toxicity of infused MAbs is expected to be low. Which enthusiastically hailed in press accounts at the time as the solution to cancer. This concept was proven by successful clinical results of mouse anti-idiotypic MAbs in the treatment of lymphomas and leukemias [1] and by FDA approval in 1986 of the OKT3 anti-CD3 mouse MAb for acute renal transplant rejection [4].

The adverse clinical and laboratory effects make the dark age of skepticism real. Therapy given by using rodent MAbs leads to the development of human anti-murine antibody (HAMA) response in more than 50\% of treated patients. Also, the effector functions of mouse antibodies have proven to be less efficient in the human context. The human immunoglobulins half-life is longer than the mouse, the factor that limits usefulness. Therefore human MAbs are theoretical solutions despite human MAbs from hybridomas and lymphocyte cell lines have proven too difficult to generate easily [5].

In 1994 the spell was broken when the FDA approved the anti-platelet mouse MAbs ReoProÔ (Centocor) to treat the complications of angioplasty [6]. Then between 1997 and 1999, FDA approved another engineered 
antibodies such as RituximabÔ, a mouse/human chimeric anti-CD2O antibody (IDEC/ Genentech, San Francisco, CA, USA) to combat follicular non-Hodgkin's B cell lymphoma [7]; ZenapaxÔ, a humanized anti-IL2 TAC receptor antibody develop by Protein Design Labs/Hoffmann- La Roche (Basel, Switzerland) to manage the acute rejection of kid [8], and HerceptinÔ, then Genentech developed the first antibody-based drug for adjuvant therapy of solid tumor (breast cancer) [9].

High target specificity and their organization into distinct structural and functional domains make antibodies preferable therapeutic candidates. When an antibody is designed as a drug, features including immunogenicity, affinity, stability, effector functions, half-life, and tissue penetration and distribution should be considered and optimized accordingly. Antibody engineering uses antibody characteristics domain and a variety of methods to develop a suited particular therapeutic use. Nineteen therapeutic antibodies have been approved by the FDA in clinical use and over 150 antibodies in clinical trials. MAbs and their recombinant derivatives are being evaluated for the prophylaxis, therapy, and control of allergic and autoimmune diseases; complications of angioplasty; sepsis; a variety of inflammatory diseases; many viral and bacterial infections; organ transplant rejection; and solid and hematological tumors [10]. Therefore, this review is organized to give insight on antibody engineering and its therapeutic application areas.

\section{GENERAL FEATURES OF ANTIBODY}

\section{Antibody Structure}

An antibody contains two light and two heavy chains, which are linked by multiple disulphide bonds (Figure 1). The light and heavy chains contain a variable region, also known as the Fab (fragment antigen binding) region, and a constant region, which is known as the Fc (fragment crystallizable) region. Also, short hypervariable amino acid sequences found in the variable domains of both light and heavy chains are known as antigen-binding complementarity determining regions (CDRs), the majority of the sequence variations associated with antibodies found in CRDs. Among the six CDRs in an IgG molecule, CDR3s have the greatest variability. In IgG, IgA, and IgD antibody isotypes, the Fc region (the tail region of antibody) is composed of two identical protein fragments, derived from the second and third constant domains of the antibody's two heavy chains. The Fc regions in IgM and IgE contain three heavy chain constant domains in each polypeptide chain (Figure 2). The IgG isotype is most commonly used in therapeutic applications [11].

\section{Functions of antibody}

Antibodies recognize specific antigens on target pathogens and have protective effects. Some of the protective effects are virus and toxin neutralization, binding of the antibody alone provides sufficient steric interference to disrupt the interaction between the cellular receptor and the antigen, thereby abrogating virus uptake and replication or intoxication. But other protective effects, such as complement activation or antibodydependent cellular cytotoxicity (ADCC) and phagocytosis activity lies in the bound antibody recruiting other components of the immune system. This occurs through Fc receptors $(F C R)$ on the surface of immune cells to the $F c$ region of the antibody or the binding of complement proteins in the serum. Direct antifungal and in vitro antibacterial effect of antibody [12] and cell-mediated immune and inflammatory response regulative characteristics [13] were found recently (Figure 3).

\section{Classification of antibodies}

There are 5 major classes of antibodies, IgM, IgG, IgA, IgD, and IgE. These immunoglobulins differ in both their structure and immune function. IgG antibodies are monomer in structure and are the dominant form of immunoglobulin found in the serum. IgM is pentameric in structure and is clinically significant because they are predominant in early immune responses to most antigens. IgAs are polymeric and are the predominant immunoglobulin in saliva, tears, nasal mucosa, prostatic fluid, and many other bodily fluids. IgD antibodies are monomeric and trace amounts are present in serum and are found on the surface of human B lymphocytes. IgE antibodies are present in serum in a monomeric form and represent only a small fraction of total antibodies in the blood. They are involved in the production and release of vasoactive mediators e.g. histamine and other chemicals that cause an inflammatory reaction. In healthy adults, the four-polypeptide chain IgG monomer constitutes approximately $75 \%$ of the total serum immunoglobulins [14] (Figure 2). 

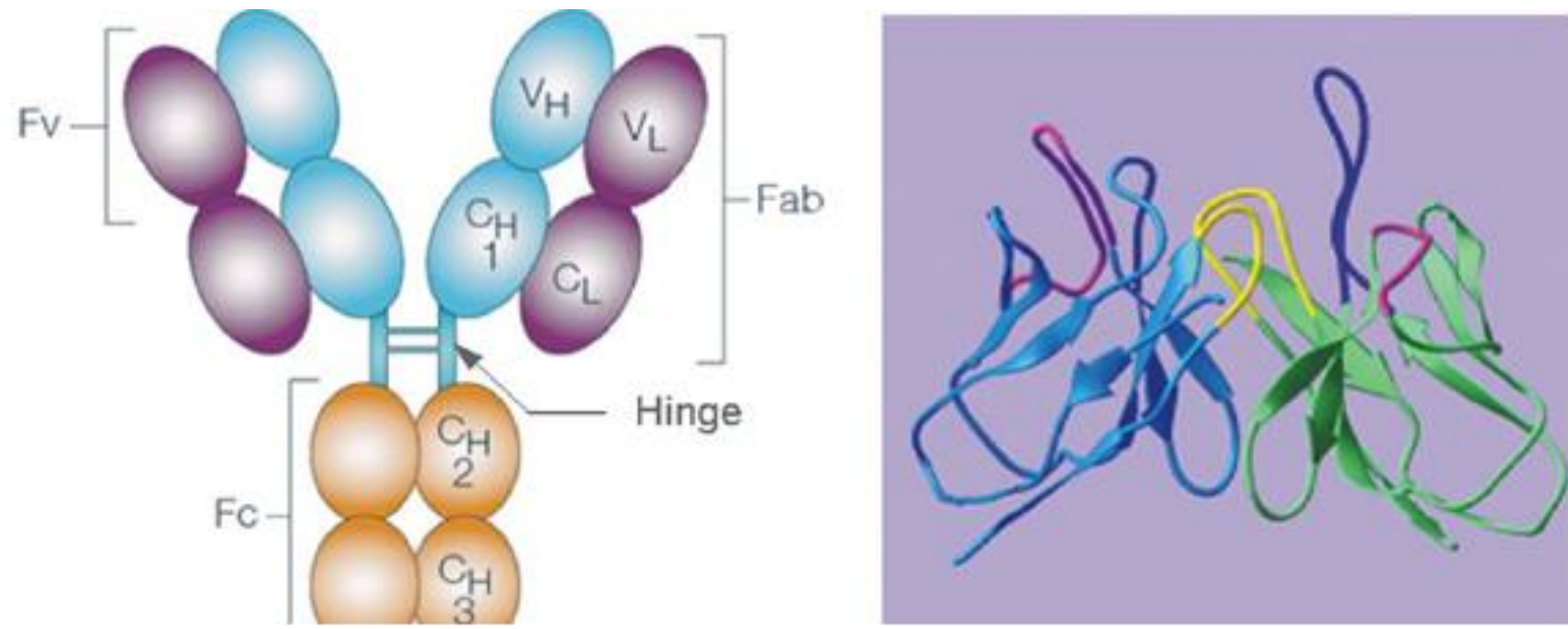

Figure 1. The modular structure of antibody molecule $(A)$ and the CDR1, 2, and 3 within the heavy and light chain variable domains (B); [10].

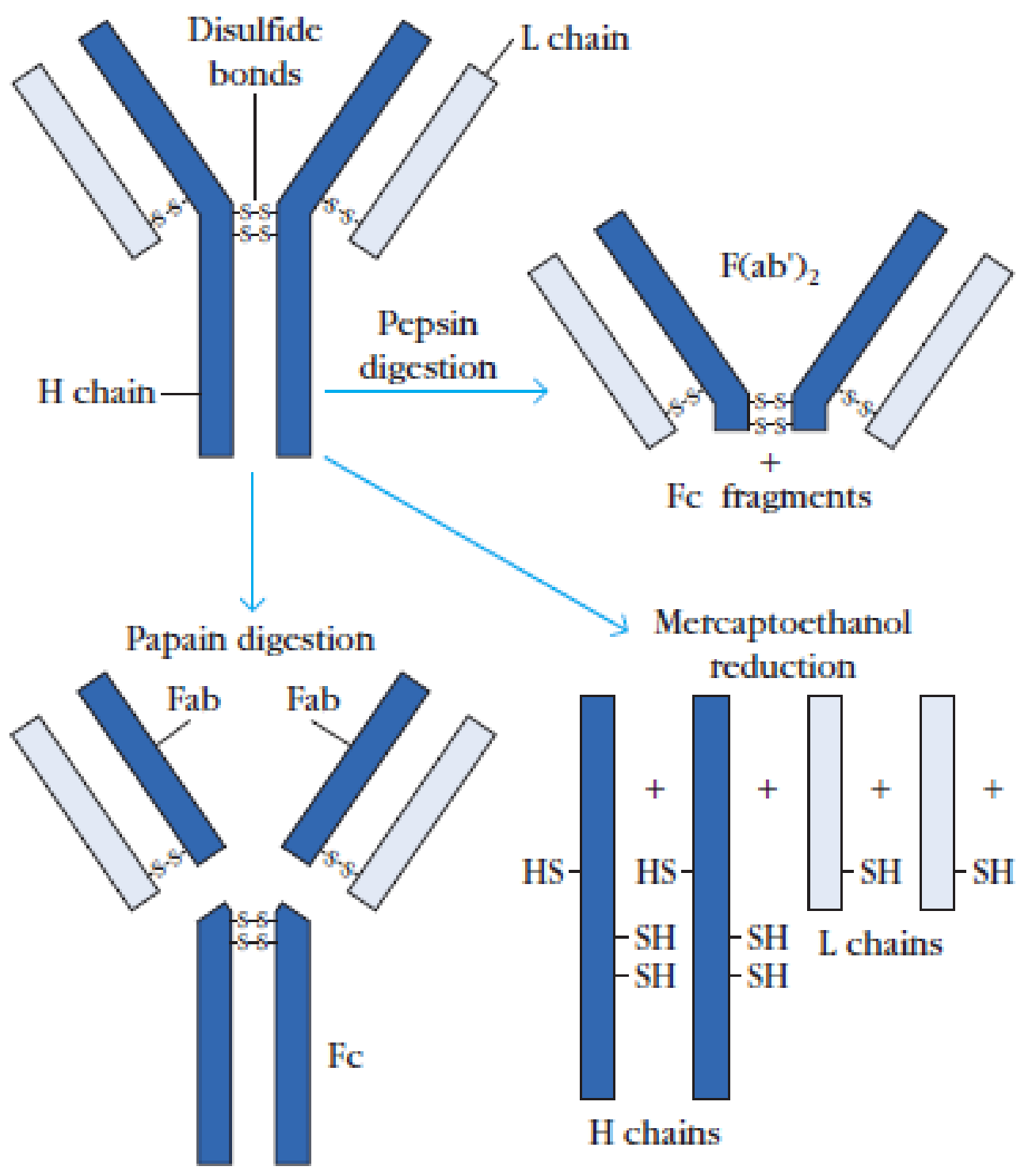

Figure 2. Prototype structure of IgG, showing chain structure, and interchain disulfide bonds. The fragments produced by enzymatic digestion with pepsin or papain or by cleavage of the disulfide bonds with mercaptoethanol are indicated; [15]. 


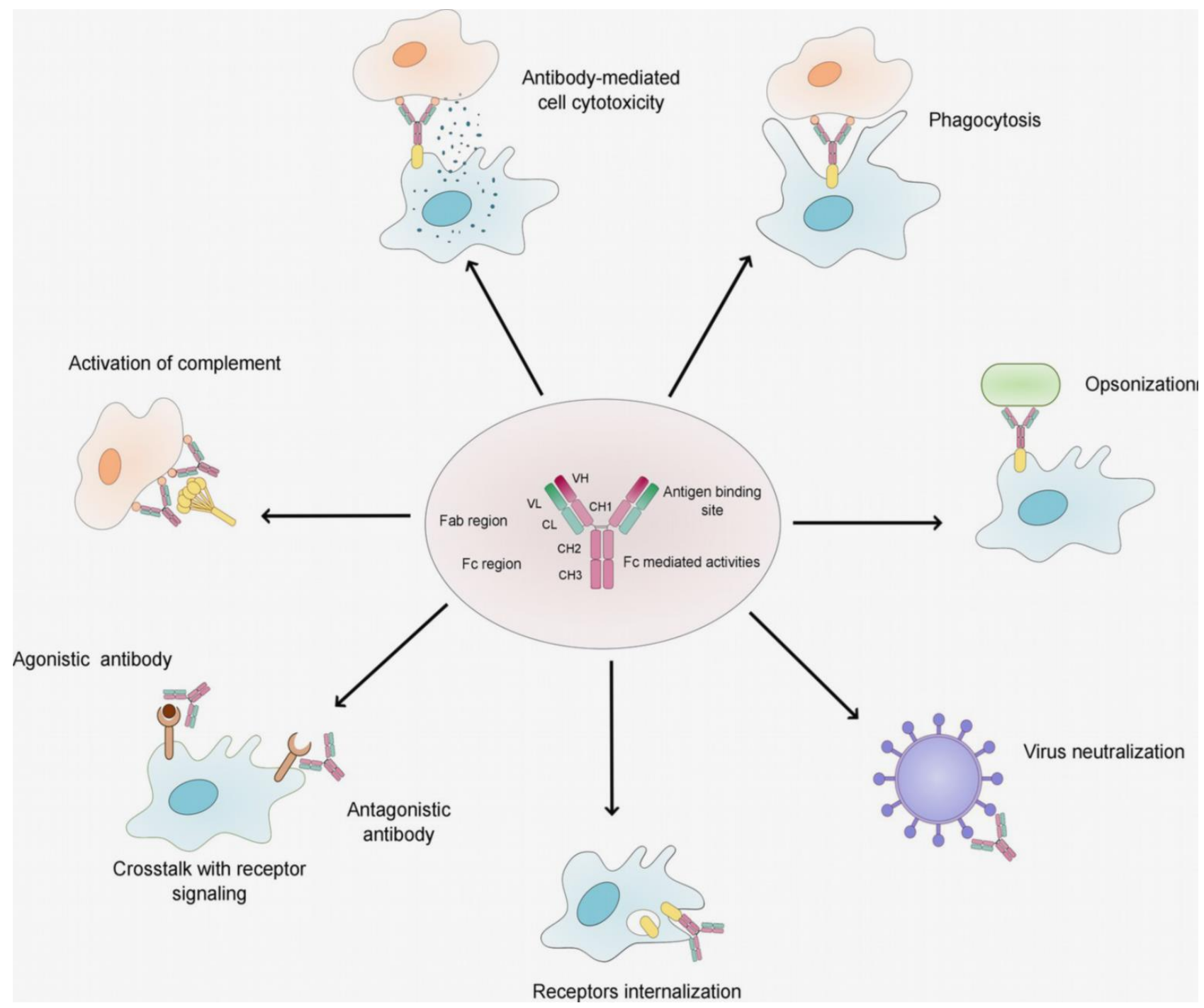

Figure 3. Overview of the natural function of antibodies; [16].

\section{ANTIBODY ENGINEERING}

\section{Monoclonal antibodies production by Hybridoma Technology}

Over a century ago Paul Ehrlich (19 ${ }^{\text {th }}$ Century) coined the phrase magic bullets to explain how antibodies might target and interact with their respective antigens. Seventy-five years later, Georges Kohler and Cesar Milstein invented the technology for cloning individual antibodies. Monoclonal antibodies specifically recognize one epitope of the cognate antigen. They can be generated using hybridoma technology which involves fusing lymphocytes from the spleen of an immunized mouse with an immortal cancer cell line (myeloma). The myeloma cell lines are selected so that they do not possess the capability to produce immunoglobulins unless fused to lymphocytes. This fusion is performed using polyethylene glycol (PEG) which is a poly wax solution that enhances adjacent cell fusions and the exchange of nuclei [17].

A mixed population of fused cells, unfused myelomas, and lymphocytes results, and these are incubated for 7 days before screening. The fused hybridoma cells are selected using HAT (Hypoxanthine, Aminopterin, and Thymidine)-containing medium. Myeloma cells lack the HGPRT (hypoxanthine-guanine phosphoribosyltransferase) enzyme and when the de novo synthesis of purine and pyrimidines is blocked by HAT addition the cells die, while lymphocytes which do not grow in this culture media eventually die. Therefore, in the presence of HAT supplemented media only the hybridoma cells will proliferate. Secreted antibodies in conditioned media from each hybridoma are tested against the immunized antigen by ELISA. Positive hybridomas are scaled up and subsequently cloned (by limiting 21 dilutions) to select for a single monoclonal antibody-producing hybridoma cell. The hybridoma cells can be stored indefinitely in liquid nitrogen [18] (Figure 4). 


\section{Hybridoma Technology}

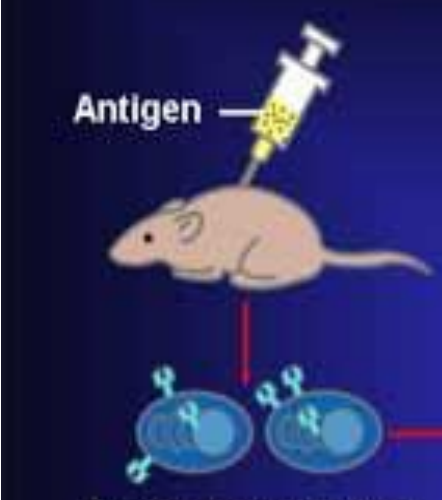

Antibody-producing

plasma cells

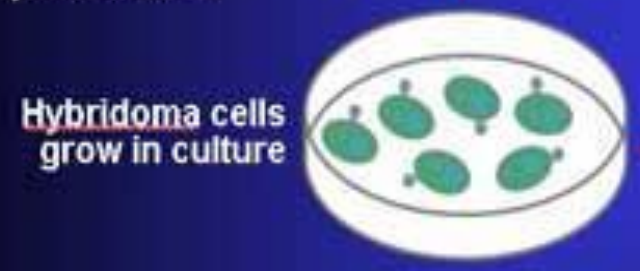

\section{Individual hybridoma cells are cloned}

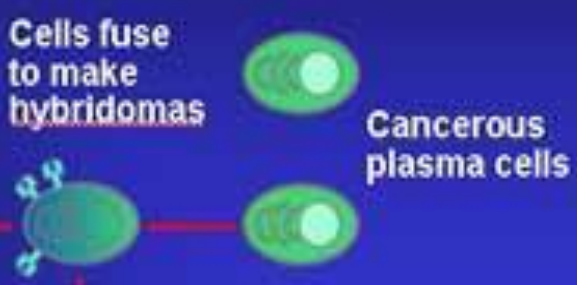

Cancerous

plasma cells

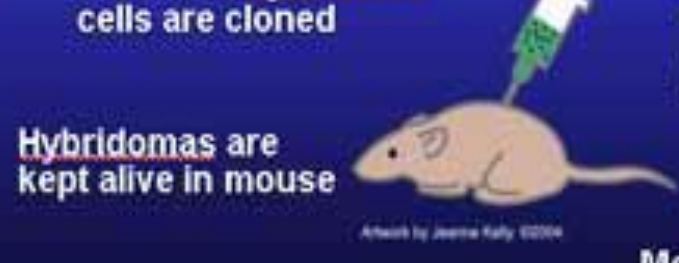

Desired clones are cultured and frozen

\section{Clones are tested for} desired antibody

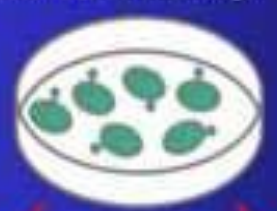

Monoclonal antibodies

are purified

Figure 4. Production of monoclonal antibody by hybridoma technology. The hybridoma technology outline involves the isolation of spleen cells from immunized mice, their fusion with immortal myeloma cells, and the production and further propagation of monoclonal antibodies from the hybrid cells; [18].

\section{Recombinant monoclonal antibodies}

Chimerization. Chimeric antibody refers to the replacement of murine constant region with equivalent human regions contributes significantly to the immunogenicity. Also, it allows for better interaction with the complement system and human effector cells (Figure 5). This strategy led to therapeutic successes such as basiliximab (Simulect: IgG1 anti-CD25, Novartis) or cetuximab (Erbitux: IgG1 anti-EGFR, ImClone) [19].

Humanization of murine antibodies. Antibody humanization technology was developed and made possible by the transfer of all xenogeneic CDRs (complementary determining regions) onto the framework of a human $\mathrm{Ab}$. The first humanized Abs was constructed based on human sequences with the known crystal structure, which permits the identification of residues contributing to the antigen-binding. In the "best fit" strategy, the closest human sequence, usually rearranged, is used as a framework to receive the murine CDRs [20] (Figure 5).

Generation of fully human monoclonal antibodies. Human antibodies have been generated by a combined polyethylene glycol/electrofusion method. Recent technologic advances allowed the production of new monoclonal antibodies Moreover; alternative approaches based on transgenic mice or in phage display were developed [21].

Human antibodies by phage display technology. Antibody phage display technology consists of the selection of antibody fragments from combinatorial libraries displayed on the surface of filamentous phage. The principle is to generate antibodies by cloning immunoglobulin variable genes using recombinant DNA technology into the phage genome. This technique allows displaying on the surface of bacteriophages the antibody fragment of interest in an scFv or Fab fragment format [22] (Figure 6). 


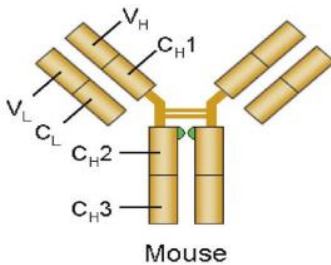

Mouse

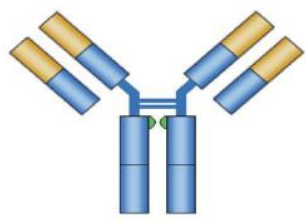

Chimeric

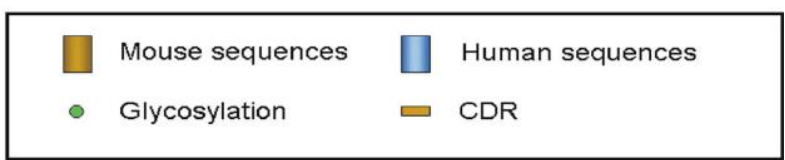

Figure 5. Antibody engineering for humanization; [10].

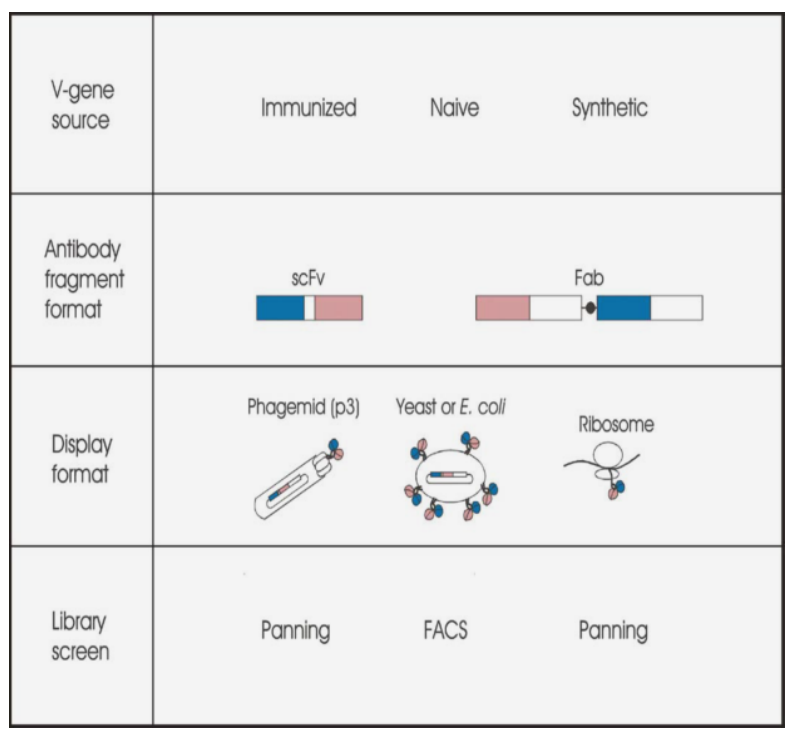

Figure 6. The display and screening system of antibody libraries; [10].

Immune library: Humans exposed to specific desired antigens through diseases or vaccination have high levels of circulating antibodies. Small libraries ( 105) from immunize are enough to give rise to specific antibodies. Due to antibody genes in vitro experienced affinity maturation, antibodies that do not require further affinity maturation can potentially be isolated. Human antibodies cannot be generated by immunization to give human MAbs because of ethical issues. Immunological tolerance makes it difficult to isolate against selfantigens, many of which are potentially important therapeutic targets, particularly in cancer [10].

Naïve library: stands for the V-gene repertoire created by cloning the antibody genes found in nonimmunized individuals. Peripheral lymphocytes, bone marrow, tonsils, and cadaver spleens are sites for mRNA isolation. From the mRNA, IgM and/or IgG variable regions are amplified by PCR using degenerate oligonucleotide primer sets and cloned into vectors suitable for screening. Subsequently, from mRNA IgM repertoires are preferred to IgG because they have not been subjected to antigen selection or tolerance they are more diverse. In contrast, IgG chains can be biased by host immune responses will not be reactive with selfantigens. Once a library has been made, it can be propagated and used repeatedly to isolate antibodies against numerous antigens. Even though, host bacteria toxicity and poor expression are often issues with antibodies isolated from naïve libraries. This condition can be circumvented by using synthetic antibody repertoire libraries [23] (Figure 5). Synthetic library: refers to fully synthetic repertoires, germline antibody gene segments, $\mathrm{VH}, \mathrm{DH}$, and $\mathrm{JH}$ or $\mathrm{V} \kappa / \lambda$ and $J \kappa / \lambda$ are cloned and arranged combinatorially in vitro to reconstitute genes encoding complete VH and VL chains. These synthetic libraries of 107-1010 clones gave rise to antibodies with specificity to self-antigens [23].

Antibody library screening is the most frequently used technique. It is based on the display of antibodies on the surface of filamentous bacteriophages. The antibody library in the Fab or scFv format is fused to a surface protein of phages, displaying antigen-specific antibodies. This can readily be enriched by selective adsorption onto immobilized antigen, a process known as panning. The bound phage is eluted from the surface and amplified through infection of E. coli cells. They are 5-8 rounds of panning, elution, and amplification are sufficient to select for phages displaying specific antibodies, even they are up to 1011 clones large libraries [24].

Cell display antibodies are antibodies that are displayed on the surface of microbial cells such as E. coli and Saccharomyces cerevisiae. A library of cells, each displaying multiple copies of a different antibody variant, is incubated with a fluorescently tagged ligand in a buffer for screening. Cells displaying antibodies become fluorescently labeled after binding with the ligand and isolated by fluorescence-activated cell sorting (FACS). The binding of each clone in the library to a particular ligand is quantified by flow cytometry. Parameters such as ligand concentration or time for the dissociation of antibody ligand complexes can be easily optimized to ensure the isolation of only the highest affinity antibodies [25]. Ribosome display stands for the formation of a ternary complex between ribosomes, mRNA, and the polypeptide. From the ribosome-mRNA-protein complexes, the mRNA is reverse transcribed to produce the DNA encoding the antibodies responsible for the binding of the complexes to the immobilized ligand. Another cycle of ternary complex formation and selection was done after transcribing the DNA by RNA polymerase [26]. 
Human antibodies from transgenic mice. The creation of transgenic mice expressing human immunoglobulin genes required two major genetic manipulations, the inactivation of the mouse antibody production machinery and the introduction of human immunoglobulin loci in their germline configuration. This complex process required the separated generation of mouse strains with inactivated mouse immunoglobulin genes or newly introduced human immunoglobulin loci and their successive crossbreeding. At the end of the process, it was possible to obtain mouse strains with a large and diverse $\mathrm{V}$ gene repertoire that, in a full immunocompetent context and upon immunization, can produce high-affinity human IgGk and IgG antibodies [27].

\section{Antibody produced by transgenic technology}

Antibodies produced in transgenic plants. In 1989 the first functional antibodies were produced from tobacco plants [28]. Independent cloning of heavy and light chain antibody genes in Agrobacterium tumefaciens vectors, the transformation of plant tissue in vitro with the recombinant bacterium, the reconstitution of whole plants expressing individual chains and their sexual cross was used to produce to generate plant antibodies. A fully assembled and functional antibody was recovered from plant tissue in some double transgenic plants found in Mendelian fashion. The progressive improvement of vectors for plantibodies, purification strategies, and the increase in transformable crop species could lead to the nearly limitless availability of inexpensive (even edible forms of) recombinant immunoglobulins for human and animal therapy and industrial applications of antibodies (e.g., catalytic antibodies) [29].

Antibodies produced in transgenic animals. In the field of recombinant immunoglobulins, there are at least three examples of the application of transgenic animal technology. The first application is related to the use of transgenic animals as bioreactors with the production of antibodies in milk. Many human proteins of therapeutic importance found in the milk of transgenic animals have been well known for years, with average yields that amount to grams per liter in different domestic species (e.g., cattle, goats, sheep, and pig) were expressed. Antibody production from mammary tissue-specific promoters with correct assembly and function of the foreign bodies in milk [30]. Creating animals that constitutively produce recombinant antibodies or antibody fragments capable of neutralizing common pathogens of the species was the second application of transgenic animal technology. The other technology was to produce mice that carry human constant and variable gene segments in germline configuration. The animal transgenesis technology uses mice produced rearranged human antibodies in their B cells and produce human antibodies after conventional immunization procedures [31].

\section{Engineering of Antibody fragments}

The whole antibody with a molecular weight of about $150 \mathrm{kDa}$ diffuses poorly from the vascular bed into a solid tumor mass and clears slowly from the body. Antibody fragments such as Fab, scFv, diabodies, and mini bodies can be generated by removing the entire constant region or part or whole of the Fc portion (Figure. 7). These antibody fragments are known to have better clearance from the whole body and tissue/tumor penetration characteristics. Antibody fragments are thought to be easy to produce in bacteria in large amounts and are therefore considered to bypass the hurdles associated with mammalian cell-based production of whole antibodies. Thus, antibody fragments are better suited for imaging and/or radiotherapy [32].

The smallest fragment of an antibody that retains the antigen-binding specificity of the whole antibody is the $\mathrm{Fv}$, in which single $\mathrm{V}$ domain binding to antigens is possible but commonly the $\mathrm{VH}$ and VL domains are noncovalently associated. Because of its instability at low concentrations, the VH and VL domains of Fv are linked by a flexible peptide linker to make a single-chain Fv (scFv) (Figure. 7B). The most common peptide linker is flexible (Gly4Ser). Also, Fv is engineered to form a disulfide bond by introducing two cysteine residues in the framework regions of VH and VL to yield a disulfide stabilized Fv (dsFv) (Figure 7C) [10].

Diabodies are scFvs homodimers are covalently linked by a short peptide linker of four amino acids which forces the $\mathrm{V}$ domains to make inter-molecular complexes with their cognate domains (Figure 7D) [33]. In a slightly different format called (dsFv) (Figure 7E), the VH and VL are engineered to form a dsFv but the VH is expressed twice in tandem separated by a flexible linker. When the VH and VL are co-expressed, a divalent molecule is formed (Figure 7F) [34]. Minibodies are scFvs-CH3 fusion protein homodimers (Figure 7G). In a different variant called Flex mini body (Figure $7 \mathrm{H}$ ), the scFv is fused to the hinge region of IgGl which in turn is fused via an additional 10 residues to the $\mathrm{CH} 3$ domain [35]. 
A

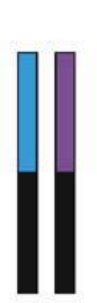

Fab
B C

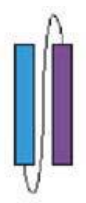

II

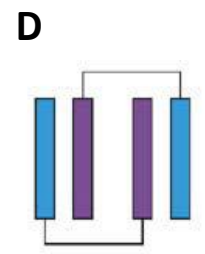

E

F

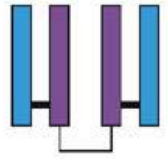

G.

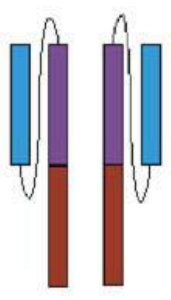

Minibody
Flex minibody
H

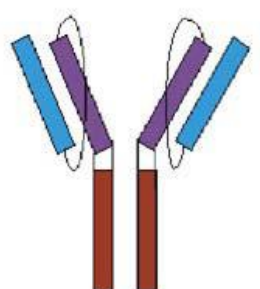

IIIII

Bi-specific

Figure 7. Schematic representation of antibody fragments; [10].

Bispecific antibodies are artificially designed molecules, capable of simultaneously binding two different antigens; hence, they can be applied to redirect effector cells to tumor cells. Technologically, bsAbs can now be produced in various ways in more than 50 formats, including quadromas, chemical conjugates, genetically linked antibody fragments, and engineered constant regions that enable heterophilic association of two heavy chains (so-called "knobs-into-holes" technology) [36], (Figure 8).

A key advantage of using bsAbs for therapy is that they make it possible to block multiple therapeutic targets with a single agent, and the effects are more pronounced when they provide pharmacological effects that a simple combination of each single-specific antibody cannot achieve. Removab (catumaxomab), the first bsAb was approved for the treatment of malignant ascites in 2009. This antibody can simultaneously bind to CD3 and EpCAM, and thus, can activate and redirect T cells to EpCAM-positive tumors [37].

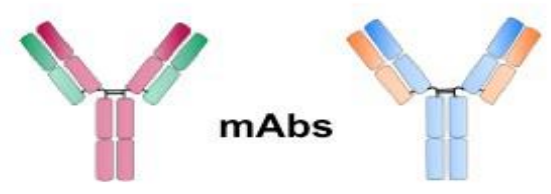

A.

B.

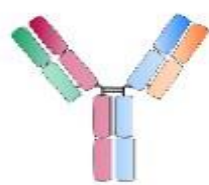

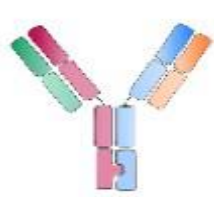

D.

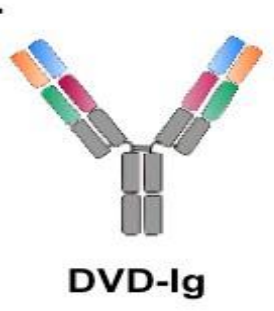

c.

$$
\text { Quadroma “Knobs-into-holes” DVD-Ig }
$$

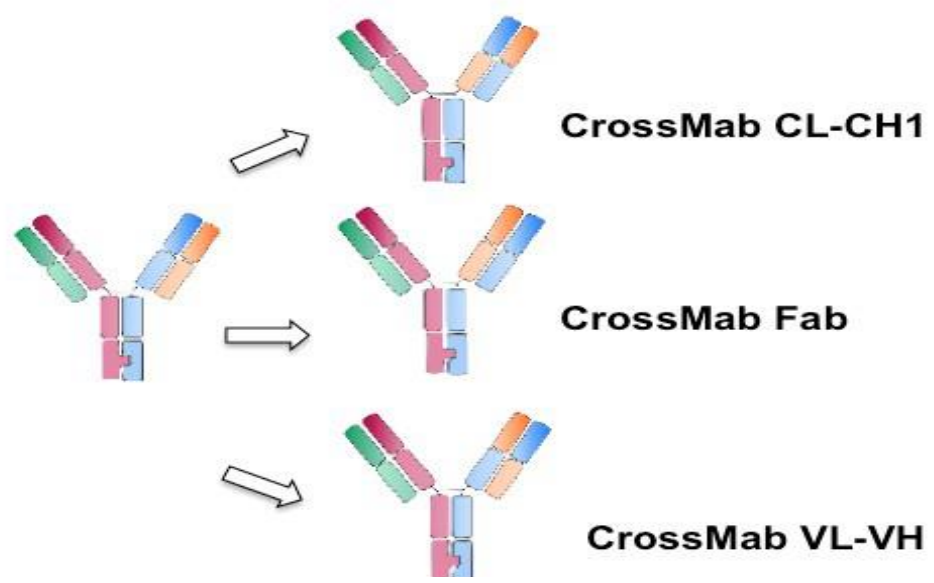

Figure 8. Schematic illustration of different formats of IgG-like bispecific antibodies with (A) Quadroma approach, (B) "Knobs-into-holes approach", (C) Cross MAb approach. (D) Dual-Variable-Domain immunoglobulin (DVD-Ig) bispecific antibody with two variable domains; [16]. 


\section{Improvement of antigen-binding affinity and effector functions}

Improvement of antigen-binding affinity. Improving the antigen-binding activity of antibodies is one of the most promising study areas in antibody engineering. There are two common approaches. Creating a very large library of randomly mutated CDRs or the entire variable domains and selecting higher affinity variants from this large collection of mutants. The large libraries are created by chain shuffling, error-prone, random CDR mutagenesis, and DNA shuffling [38]. The other approach is focused on or hot spot mutagenesis mimicking in vivo affinity maturation in small libraries [39].

Improvement of effector functions. To improve effector functions therapeutic antibodies follow two basic mechanisms. The first mechanism is triggering intracellular signals. In this mechanism, the antibodies are largely dependent on their antigen-binding function rather than effector function in apoptosis and blocking ligand interaction. Recruiting immune system components following antigen-binding ability as well as their ability to trigger effector activity is the second mechanism of therapeutic antibodies. Antigen binding ability and engineering antibodies improve antibody binding to Fc $\gamma$ Rs or the complement factors determine the therapeutic efficacy of the antibodies. Glycoengineering of IgG Fc and mutating the residues that contribute to FcyR binding which improves ADCC activity [40]. The molecules of Clqs to the hinge and $\mathrm{CH} 2$ domain of antibodies in which the CDC depends on. Employing domain switching and site-directed mutagenesis of IgG constant domains indicated that the $\mathrm{CH} 2$ domain had an important contribution to the $\mathrm{CDC}$ [10].

\section{Altering pharmacokinetics}

Increased FcRn binding. FcRn receptor binding with a mapped and well-characterized site on IgG determines the plasma half-life of IgGl. Binding to $\mathrm{FcRn}$ is increased by mutating the FcRn binding site such that More recently two mutations, T250Q and M428L, have been discovered that caused respectively a 3- and a 7-fold increase in FcRn binding and when combined together caused a 28-fold increase in FcRn binding [41].

PEGylation. An increase in the half-lives of antibody fragments is an important area of research in the development of the methods. PEGylation of proteins and liposomes has been a time tested and successful technique that offered the advantage of reducing immunogenicity, increasing the plasma half-life, increasing solubility, and reducing protease sensitivity. This process is conjugating polyethylene glycol chains to the antibody fragments. Prolongation of the phase, a phase that represents the redistribution of a molecule in the extravascular environment increase in half-life observed with PEGylated antibody fragments. PEGylation slows the redistribution of the molecules from the plasma to the interstitial compartment. Therefore, the science of antibody PEGylation has two focus areas (a) to preserve the antigen-binding activity completely and (b) to link the PEG molecule to the antibody in a stable manner [42].

\section{Other antibody constructions}

Antibodies can be produced genetic fusion proteins with toxins, drugs, enzymes, and other functional groups and modified in their constant domains to alter the original effector mechanisms and properties of the antibody molecule [43]. Immunoadhesins are fusion proteins that combine the hinge and Fc regions of an antibody with domains of a ligand-specific cell surface receptor. These molecules are used as laboratory experimental tools and as promising applications in medicine [44]. Antigenized antibodies are made up of peptide epitopes derived from antigens that are different from immunoglobulins in the place of the CDR loops of immunoglobulin by grafting. The conformationally restricted exposure of short foreign peptides using the $\mathrm{V}$ region framework and the characteristics of the constant antibody domains create promising combinations for immunoprophylaxis or immunotherapy. They can also be extended to the peptide hormone field and the rational design of new drugs [43].

\section{APPLICATION OF ENGINEERED ANTIBODIES FOR THERAPEUTIC PURPOSES}

\section{Antibody engineering as an opportunity for selection and optimization of anti-HIV therapeutic agents}

Clinical need for new therapeutic approaches

Human Immunodeficiency Virus type 1 (HIV-1) is the causal agent of the acquired immunodeficiency syndrome (AIDS). The introduction of highly active antiretroviral therapy (HAART), has greatly improved the quality of life of many infected individuals, and mortality for AIDS has dropped dramatically. However, currently available antiretroviral drugs have three major shortfalls: 1) the drugs have relatively high toxicities that cause undesirable side effects, including myocardial infarction; 2) the drug activity of the Reverse 
Transcriptase inhibitors is cell-dependent since they are active post-infection, and 3) HIV has a high mutation rate leading to the rapid development of drug-resistant viral variants. For these reasons, many patients treated with HAART regimens still fail to achieve or maintain optimal control of the infection and development of HIV-1 drug resistance due to the failure of available Anti-retroviral drugs. Therefore, other methods of intervention through control viral replication are needed. Drugs that target the cellular receptor complex are promising areas in HIV therapy and are a good area of interest for multi-drug resistant viruses [45].

Anti-HIV antibodies currently in clinical trial. The major characteristics of the antibodies already entered in clinical trials as HIV entry inhibitors are summarized in Table 1. The monoclonal antibody Ibalizumab, formerly named TNX-355 and Hu5A8, is a humanized IgG4 mAb that binds to the second (C2) domain of CD4. In contrast to attachment inhibitors, Ibalizumab decreases the flexibility of CD4, thereby hindering the access of the CD4gp120 complex to the co-receptors CCR5 and CXCR4 rather than preventing gp12o binding to CD4. This mAb is a potent inhibitor of HIV-1 in vitro and shows synergy when combined with other anti-HIV drugs or the fusion inhibitor enfuvirtide [46].

Table 1. Antibodies who have Completed Clinical Trials for HIV

\begin{tabular}{lccccc}
\hline $\begin{array}{l}\text { Antibody } \\
\text { Name }\end{array}$ & Target Ag & Origin & Isotype & Trial status & References \\
\hline Ibalizumab & CD4 & Humanized mAb & IgG4 & II & {$[46]$} \\
PRO542 & Gp120 & Human fusion protein & IgG2 & II & {$[47]$} \\
PRO140 & CCR5 & Humanized mAb & IgG4 & I & {$[48]$} \\
HGS004 & CCR5 & Human mAb & IgG4 & I & {$[49]$} \\
\hline
\end{tabular}

\section{Antibody therapeutics for cancer therapy}

Monoclonal Antibodies (mAbs) comprise a class of therapeutic biologics that have been increasingly used over the last decades. The concept of using antibodies to selectively target tumors was proposed by Paul Ehrlich over a century ago [50].

\section{Mechanism of action of monoclonal antibodies for the treatment of cancer}

Altering signal transduction in the downstream intracellular pathways. Various cell surface receptors that activate intracellular pathways are expressed by cancer cells leading to growth. Amongst these, EGFR or ErbBl, ErbB2 or HER-2/Neu, HER-3, and HER-4 are of the same family and are over-expressed in epithelial malignancies originating from the colon, breast, lung, and head and neck resulting in rapidly proliferating disease and increased metastatic potential. Downstream activation of the receptor and increasing receptor internalization inhibited by Anti-EGFR antibodies bind to the receptor domain of the EGFR receptor. The cancer cell cycle inhibited by antibodies and cause apoptosis [51].

Antibody-dependent cell cytotoxicity (ADCC). The cancer cells that are coated by antibodies resulted in immune-mediated destruction. The effector cells in the antibody-dependent cytotoxicity include macrophages, NK cells, and neutrophils. ADCC depends on the Fc portion of the antibody that binds an Fc gamma receptor (FcgR) on the effector cells. ADCC occurs when the Fab and Fc portions of the mAb engage both tumor cell antigen and an activating FcgR, respectively, thus creating a bridge from the tumor cell to the effector cell. Target cell recognition is then coupled to a lytic attack on the target cell mounted by effector cells [52].

Complement-mediated cytotoxicity (CDC). CDC results from a cytolytic cascade mediated by a series of complement proteins, resulting in lysis of the antibody-bound cell [51].

Soluble ligand neutralization. Antibodies can bind to circulating proteins and interfere with their ability to find their targets to help facilitate the growth of the tumors. One important example of this mechanism is bevacizumab which is a fully-humanized monoclonal antibody against VEGF-A. Bevacizumab binds and inactivates the biological activity of VEGF-A, inhibiting angiogenesis, and thus, tumor growth and proliferation [53].

Cytotoxic drug delivery. Tumor-targeted monoclonal antibodies are linked to Cytotoxic agents to deliver them specifically to the tumor cells which is preferable for its limited systemic side effect [54]. Two exciting samples of this technology are trastuzumab-DM1 T-DMl, a HER2 directed antibody-drug conjugate, and brentuximab vedotin, a CD30 directed antibody-drug conjugate [51]. Few antibody therapies are developed for cancer in animal besides human cancer therapy. for instance, Aratana Therapeutics, a pet health company 
based in Kansas City, USA is developing antibody therapies for canines. Canine lymphoma monoclonal antibody- AT-005 (against CD52) has been approved in 2012, for the treatment of T-cell lymphoma in dogs [55].

Monoclonal antibodies approved for clinical use in oncology. The major characteristics of monoclonal antibodies approved for oncology therapy are summarized in Table 2.

Table 2. Monoclonal antibodies approved for clinical use in oncology

\section{The use of antibodies in the treatment of infectious diseases}

Anti-bacterial antibodies. Antibiotic therapy targets bacterial exotoxins. for instance, B. anthracis produces a potent bioweapon, a tripartite exotoxin consisting of a protective antigen (PA), lethal factor (LF), and edema factor (EF). Post-exposure prophylaxis with $\mathrm{mAb}$ against PA protected against a lethal inhalational anthrax challenge in rabbits and monkeys and a mAb against LF protected rats against a challenge with a lethal toxin, a mixture of PA and LF [57]. An anti-PA mAb acts synergistically with the antibiotic ciprofloxacin for cover against inhalational anthrax [58].

Escherichia coli, Shiga toxin-producing significant exotoxin-producing pathogen causes severe gastrointestinal disease. Complications like hemolytic uraemic syndrome, acute kidney failure, and death may result from toxin entry into the bloodstream. Currently, only supportive treatments are available. a human IgG1 $\mathrm{mAb}$ generated in transgenic mice against Shiga toxin subunit A prevented fatal systemic complications in piglets following administration after the onset of diarrhea [59]. Examples of other exotoxins against which mAbs are shown to possess some efficacy include Pseudomonas aeruginosa exotoxin A, eubacteria Clostridium perfringens epsilon toxin, and Clostridium botulinus neurotoxin [60]. Even though targeting of exotoxin requires prior knowledge of the pathology of the infectious agent and initial characterization of the exotoxin. Therefore the genetic nature, surface carbohydrates regions that show relatively little variability between bacteria subtypes and are explored as potential target antigens. Antibody targets against shared or invariant epitopes like the core carbohydrate backbone, as many bacterial species often exhibit variability in their carbohydrate side-chain residues. Also, targets preventing septic shock by promoting the clearance of LPS endotoxin within the bloodstream. Carbohydrates targeting mAbs mixed results and MAbs raised against the inner core LPS of varied Neisseria meningitides serotypes have shown poor phagocytic activity despite their avidity for whole-cell bacteria and showed poor binding to full-length LPS. MAbs produced against the deacetylated core carbohydrate backbone of the $S$. aureus surface carbohydrate, poly-N-acetylglucosamine (PNAG), conferred protection from a bacterial challenge in mice and performed better than mAbs against a totally acetylated wildtype PNAG [61].

In another study, mAbs raised against Streptococcus pneumoniae serotype 6B capsular polysaccharide with strong cross-reactivity for serotype 6A showed avidity-dependent in vitro opsonization and in vivo protection against a bacterial challenge with either subtype. Thus, while a generic target for bacteria is attractive, much 
work remains needed for it to be applied within the clinical setting [62]. Treatment of antibiotic-resistant bacteria, such as $S$. aureus is another area of interest during which an antibody that has reached the clinical test stage is tefibazumab (Aurexis), a humanized mAb that binds clumping factor A (ClfA), a serious virulence determinant in S. aureus. Tefibazumab has been shown to induce phagocytosis of ClfA-coated beads by human polymorphonuclear cells in vitro, protect against an intravenous challenge with $S$. aureus during a rabbit model of infective endocarditis and enhance the efficacy of vancomycin therapy within the rabbit therapeutic model [63].

Antibodies and viral disease. Palivizumab, the sole mAb currently on the marketplace for the treatment of infectious diseases, was developed as a prophylactic treatment against the viral disease Respiratory Syncytial Virus (RSV). Although mAbs are shown to be ready to neutralize many viral pathogens in vitro, the utility of $\mathrm{mAb}$ therapy in viral diseases remains a matter of contention because it is unclear to what extent viral clearance depends on antibody-mediated immunity. The clearance of a virus infection is typically related to $\mathrm{T}$ cell-mediated adaptive immunity. CD8+ $\mathrm{T}$ cells act by killing virus-infected cells, thus preventing viral replication and reducing the viral load. However, in acute infections, neutralizing therapeutic antibodies should be ready to help by suppressing viral replication and viremia, giving the host system time to develop an efficient response for viral clearance. Additionally, antibodies can promote the killing of infected cells expressing viral proteins on their surface through the activation of natural killer (NK) cells that mediate ADCC, additionally to their viral neutralization properties [64].

For viral infections where the host system is unable to completely clear the virus, resulting in chronic infection, the administration of neutralizing antibodies might not be ready to achieve complete clearance. In two separate clinical trials using human mAbs against a hepatitis B virus $S$ antigen to treat patients with chronic hepatitis B infection, the viral DNA and S antigen load in serum were significantly and rapidly reduced after antibody administration. It has been also shown the upkeep of a 90\% reduction in S antigen levels 15 days after cessation of therapy in half the patients, and this correlated with the persistence of the administered mAb in serum. However, in both studies, DNA and S antigen levels eventually recovered once antibody levels within the serum declined following the cessation of therapy. Nonetheless, regular administration of therapeutic antibodies should prove useful by preventing disease transmission, the infection of healthy cells, or the event of pathology through the continued suppression of viral levels [65].

\section{LIMITATIONS OF ANTIBODY ENGINEERING}

\section{Production costs}

Monoclonal antibodies are large $(150 \mathrm{kDa})$ multimeric proteins containing numerous disulphide bonds and post-translational modifications like glycosylation. They have classy eukaryotic machinery to be produced in active form. Moreover, most studies have shown that these molecules need to be injected in large amounts to realize clinical efficacy (e.g. 8-16 doses of $375 \mathrm{mg} \cdot \mathrm{m}-2$, that is, a complete amount of 6-12 $\mathrm{g}$ per patient for Rituximab. Consequently, the assembly of therapeutic antibodies necessitates extensive purification steps; under good manufacturing practice conditions, resulting in extremely high production costs and limiting the wide use of those drugs [66].

\section{Pharmacokinetics versus tissue penetration}

Monoclonal antibodies against tumor-specific antigens only $20 \%$ of the administered dose typically interacts with the tumor cells in murine xenograft models. Largely remain in the blood is the major limitation observed [67]. Favorable pharmacokinetics and efficient penetration and retention in the targeted tissue, and various characteristics of mAbs, such as molecular size, shape, affinity, and valency determine tumor cell antibody uptake. The large size of the monoclonal antibody prevents them from the renal clearance threshold $(\sim 70 \mathrm{kDa})$, preventing them from being eliminated through the kidney glomeruli and the half-life is prolonged. Fc portion of IgG molecules can interact with various receptors expressed at the surface of several cell types, which increase their retention in the circulation. Moreover, the Fc portion of IgG molecules can interact with various receptors expressed at the surface of several cell types, which increases their retention in the circulation. Most importantly, the Fc portion can interact with the neonatal Fc receptor (FcRn) expressed at the surface of several cell types, including vascular endothelium cells, monocytes, and macrophages as well as with barrier sites such as the blood-brain interface, the glomerular filter in the kidneys and the intestinal epithelium [68]. 


\section{Mode of action and associated limitations}

Binding of the antibody to its antigen, thereby interfering with its activity and interaction with binding partners is the simplest way MAbs to function. ADCC plays a major role in the in vivo efficacy of mAbs. However, they trigger ADCC by therapeutic antibodies that have several limitations. The first limitation arises from, $80 \%$ of the population expresses a low-affinity variant of the receptor as a major issue and the affinity between the Fc and its receptors plays a crucial role. The other one that arises from IgGi molecules are glycosylated in the $\mathrm{CH} 2$ domain (Asn 297) of the Fc region. Modification is extremely important as it modulates the affinity of the Fc for FcyRIIIa, thereby modifying the in vivo efficacy of antibodies. The third limitation because of the competition of therapeutic antibodies with a high concentration of patient's IgGs for binding to Fc $\gamma$ RIIIa. Even though, $66 \%$ of those molecules and the serum concentration of IgG is $8-17 \mathrm{mg} \cdot \mathrm{mL}^{-1}$ capable of interacting with Fc $\gamma$ RIIIa are IgGl. To get the therapeutic effect and to reach a serum concentration between 10 and $100 \mu \mathrm{g} \cdot \mathrm{mL}^{-1}$ much high doses of an antibody are required. Whereas, $10 \mathrm{ng} \cdot \mathrm{mL}^{-1}$ in vitro is the level of antibodies saturating $A D C C$, in the absence of competing IgGs [69].

\section{CONCLUSION}

Antibodies are an extremely versatile class of antimicrobial proteins that are effective against a variety of diverse microorganisms. Modern biotechnology by using a variety of in vivo and in vitro screening methods enables researchers to produce fully human antibodies against a specific target. Antibody engineering is applied in the therapeutic industry to improve the current therapeutic nature of antibodies so that they will be effective in the treatment of different diseases. Hybridoma technology, recombinant antibody fragments, Transgenic and phage technology, and improvement of effector functions are some of the technologies that are applied to antibodies to improve their therapeutic capability. Genetic engineering can be used to harness and to reformat individual antibody obtained from hybridoma, transgenic mouse, or phage selection. Currently, diseases that are global issues such as Cancer, HIV-AIDS, and many infectious bacterial and viral livestock diseases are being treated using this new technology. Relatively expensiveness to manufacture, the requirement of systemic administration, and specificity to a particular pathogen or serotype are among the challenges in the application of antibody therapeutics. Besides researches are ongoing to improve the efficacy of antibody, to reduce the cost of production and improving affinity and specificity, with consider able success. Therefore, further investigation is needed on antibody engineering to extend the application beyond therapy. Furthermore, the technology for the antibody production should be adopted in developing countries since it is less labor demanding and used to treat a wide range of infectious agents.

\section{DECLARATIONS}

\section{Authors' contributions}

All authors contributed equally to this review.

\section{Conflicts of interest}

The Authors declare no conflict of interest.

\section{REFERENCES}

1. Gavilondo JVandLarrick JW. Antibody engineering at the millennium. Biotechniques. 2000; 29 (1): 128-145. Link Retrieved from journal.https://www.future-science.com/doi/abs/10.2144/002910vo1

2. Casadevall A. Antibody-based therapies for emerging infectious diseases. Emerging infectious diseases. 1996; 2 (3): 200. Link Retrieved from journal.https://doi.10.3201/eido203.960306

3. Ferguson $M$, Minor PD, Garrett AJ, Page $M$, Thorpe $R$, et al. Testing plasma pools for markers of viral contamination: The uk experience. Vox sanguinis. 1996; 71 (1): 21-26. Link Retrieved from journal.https://doi.org/10.1046/j.1423-0410.1996.7110021.x

4. Shield C, Jacobs J, Wyant SandDas A. A cost-effectiveness analysis of okt3 induction therapy in cadaveric kidney transplantation. Am. J. Kidney Dis. 1996; 27 (855-864). Link Retrieved from journal.https://doi.org/10.1016/So272-6386(96)90524-7

5. Dueñas $M$, Chin LT, Malmborg AC, Casalvilla $R$, Ohlin $M$, et al. In vitro immunization of naive human b cells yields high affinity immunoglobulin $\mathrm{g}$ antibodies as illustrated by phage display. Immunology. 1996; 89 (1): 1-7. Link Retrieved from journal.https://doi.org/10.1046/j.1365-2567.1996.d01-708.x

6. Glaser V. Can reopro repolish tarnished monoclonal therapeutics? Nature biotechnology. 1996; 14 (10): 1216-1217. Link Retrieved from journal.https://doi.org/10.1038/nbt10g6-1216b 
7. Grillo-López AJ, White CA, Varns C, Shen D, Wei A, et al. Overview of the clinical development of rituximab: First monoclonal antibody approved for the treatment of lymphoma. Secondary title; 1999. p. 66-73.

8. Ekberg H, Bäckman L, Tufveson GandTyden G. Zenapax (daclizumab) reduces the incidence of acute rejection episodes and improves patient survival following renal transplantation. Secondary title; 1999. p. 267-268.

9. Weiner L. Monoclonal antibody therapy of cancer. Sem. Oncol. 1999; 26: 43-51. Link Retrieved from journal

10. Kim SJ, Park YandHong HJ. Antibody engineering for the development of therapeutic antibodies. Molecules \& Cells (Springer Science \& Business Media BV). 2005; 20 (1): 17-29. Link Retrieved from journal.https://www.researchgate.net/profile/Sang_Jick_Kim/publication/7509228

11. Zhiqiang A. Antibody therapeutics-a mini review. Trends in Bio/Pharmaceutical Industry. 2008; 2: 24-29. Link Retrieved from journal

12. Connolly SE, Thanassi DGandBenach JL. Generation of a complement-independent bactericidal igm against a relapsing fever borrelia. The Journal of Immunology. 2004; 172 (2): 1191-1197. Link Retrieved from journal.https://doi.org/10.4049/jimmunol.172.2.1191

13. Casadevall AandPirofski L-a. New concepts in antibody-mediated immunity. Infection and immunity. 2004; 72 (11): 6191-6196. Link Retrieved from journal.https://doi.10.1128/IAI.72.11.6191-6196.2004

14. Mahabeer RandBhoola KD. Kallikrein and kinin receptor genes. Pharmacology \& Therapeutics. 2000; 88 (1): 77-89. Link Retrieved from journal.https://doi.org/10.1016/S0163-7258(00)00080-2

15. Owen J, Punt JandStranford S. Kuby immunology. 2013. New York: WH Freeman and Company;

16. Spasevska I. An outlook on bispecific antibodies: Methods of production and therapeutic benefits. Biosci. Master Rev. 2013; 4: 159. Link Retrieved from journal

17. Little M, Kipriyanov S, Le Gall FandMoldenhauer G. Of mice and men: Hybridoma and recombinant antibodies. Immunology today. 2000; 21 (8): 364-370. Link Retrieved from journal.https://doi.org/10.1016/So167-5699(00)01668-6

18. Pandey S. Hybridoma technology for production of monoclonal antibodies. Hybridoma. 2010; 1 (2): o17. Link Retrieved from journal.www.globalresearchonline.net

19. Baert F, Noman M, Vermeire S, Van Assche G, D'Haens G, et al. Influence of immunogenicity on the long-term efficacy of infliximab in crohn's disease. New England Journal of Medicine. 2003; 348 (7): 601-608. Link Retrieved from journal.https://www.nejm.org/doi/full/10.1056/nejmoa020888

20. Yazaki J, Sherman A, Shively E, Ikle D, Williams E, et al. Humanization of the anti-cea t84 antibody-based on crystal structure data. . Protein Eng. Des. Sel. 2004; 1: 481-489. Link Retrieved from journal

21. Kwong PD, Mascola JRandNabel GJ. Mining the b cell repertoire for broadly neutralizing monoclonal antibodies to hiv-1. Cell host \& microbe. 2009; 6 (4): 292-294. Link Retrieved from journal.https://doi.org/10.1016/j.chom.2009.09.008

22. Hoogenboom HR. Selecting and screening recombinant antibody libraries. Nature biotechnology. 2005; 23 (9): 1105-1116. Link Retrieved from journal.https://doi.org/10.1038/nbt1126

23. Griffiths ADandDuncan AR. Strategies for selection of antibodies by phage display. Current opinion in biotechnology. 1998; 9 (1): 102108. Link Retrieved from journal.https://doi.org/10.1016/S0958-1669(98)80092-X

24. Hoogenboom HR, de Brume AP, Hufton SE, Hoet RM, Arends J-W, et al. Antibody phage display technology and its applications. Immunotechnology. 1998; 4 (1): 1-20. Link Retrieved from journal.https://doi.org/10.1016/S1380-2933(98)00007-4

25. Boder ETandWittrup KD. Yeast surface display for screening combinatorial polypeptide libraries. Nature biotechnology. 1997; 15 (6): 553-557. Link Retrieved from journal.https://doi.org/10.1038/nbt0697-553

26. Hanes JandPlückthun A. In vitro selection methods for screening of peptide and protein libraries. Book title: Springer; 1999.p. 107-122.

27. Lonberg N. Human antibodies from transgenic animals. Nature biotechnology. 2005; 23 (9): 1117-1125. Link Retrieved from journal.https://doi.org/10.1038/nbt1135

28. Hiatt A, Caffferkey RandBowdish K. Production of antibodies in transgenic plants. Nature. 1989; 342 (6245): 76-78. Link Retrieved from journal.https://doi.10.1038/342076aO

29. Hendy S, Chen ZC, Barker H, Santa Cruz S, Chapman S, et al. Rapid production of single-chain fv fragments in plants using a potato virus $\mathrm{x}$ episomal vector. Journal of immunological methods. 1999; 231 (1-2): 137-146. Link Retrieved from journal.https://doi.org/10.1016/S0022-1759(99)00150-7

30. Pollock DP, Kutzko JP, Birck-Wilson E, Williams JL, Echelard Y, et al. Transgenic milk as a method for the production of recombinant antibodies. Journal of immunological methods. 1999; 231 (1-2): 147-157. Link Retrieved from journal.https://doi.org/10.1016/S0022$1759(99) 00151-9$

31. Green LL. Antibody engineering via genetic engineering of the mouse: Xenomouse strains are a vehicle for the facile generation of therapeutic human monoclonal antibodies. Journal of immunological methods. 1999; 231 (1-2): 11-23. Link Retrieved from journal.https://doi.org/10.1016/S0022-1759(99)00137-4

32. Wu A. Engineering multivalent antibody fragments for in vivo targeting. Methods Mol. Biol. 2004; 248: 209-225. Link Retrieved from journal

33. Holliger P, Prospero TandWinter G. "Diabodies": Small bivalent and bispecific antibody fragments. Proceedings of the National Academy of Sciences. 1993; 90 (14): 6444-6448. Link Retrieved from journal.https://doi.org/10.1073/pnas.90.14.6444

34. Bera TK, Onda M, Brinkmann UandPastan I. A bivalent disulfide-stabilized fv with improved antigen binding to erbb2. Journal of molecular biology. 1998; 281 (3): 475-483. Link Retrieved from journal.https://doi.org/10.1006/jmbi.1998.1948

35. Hu S-z, Shively L, Raubitschek A, Sherman M, Williams LE, et al. Minibody: A novel engineered anti-carcinoembryonic antigen antibody fragment (single-chain fv-ch3) which exhibits rapid, high-level targeting of xenografts. Cancer research. 1996; 56 (13): 3055 3061. Link Retrieved from journal.https://cancerres.aacrjournals.org/content/56/13/3055

36. Merchant AM, Zhu Z, Yuan JQ, Goddard A, Adams CW, et al. An efficient route to human bispecific igg. Nature biotechnology. $1998 ; 16$ (7): 677-681. Link Retrieved from journal.https://doi.org/10.1038/nbto798-677

37. Bokemeyer C. Catumaxomab-trifunctional anti-epcam antibody used to treat malignant ascites. Expert opinion on biological therapy. 2010; 10 (8): 1259-1269. Link Retrieved from journal.https://doi.org/10.1517/14712598.2010.504706

38. Zhang M, Shu Y, Rudolph D, Prabakaran PandLabrijn A. Improved breadth and potency of an hiv-1- neutralizing human single-chain antibody by random mutagenesis and sequential antigen panning. J. Mol. Biol. 2004; 335: 209-219. Link Retrieved from journal

39. Ho M, Kreitman RJ, Onda MandPastan I. In vitro antibody evolution targeting germline hot spots to increase activity of an anti-cd22 immunotoxin. Journal of Biological Chemistry. 2005; 280 (1): 607-617. Link Retrieved from journal.https://doi:10.1074/jbc.M409783200 
40. Daeron M. Fc receptor biology. Ann. Rev. Immunol. 1997; 15: 203-234. Link Retrieved from journal

41. Dall'Acqua WF, Woods RM, Ward ES, Palaszynski SR, Patel NK, et al. Increasing the affinity of a human iggl for the neonatal fc receptor: Biological consequences. The Journal of Immunology. 2002; 169 (9): 5171-5180. Link Retrieved from journal.https://doi.org/10.4049/jimmunol.169.9.5171

42. Chapman AP. Pegylated antibodies and antibody fragments for improved therapy: A review. Advanced drug delivery reviews. $2002 ; 54$ (4): 531-545. Link Retrieved from journal.https://doi.org/10.1016/So169-409X(02)00026-1

43. Penichet ML, Kang Y-S, Pardridge WM, Morrison SLandShin S-U. An antibody-avidin fusion protein specific for the transferrin receptor serves as a delivery vehicle for effective brain targeting: Initial applications in anti-hiv antisense drug delivery to the brain. The Journal of Immunology. 1999; 163 (8): 4421-4426. Link Retrieved from journal.https://www.jimmunol.org/content/163/8/4421

44. Chamow SMandAshkenazi A. Immunoadhesins: Principles and applications. Trends in biotechnology. 1996; 14 (2): 52-60. Link Retrieved from journal.https://www.cell.com/trends/biotechnology/pdf/0167-7799(96)80921-8.pdf

45. Hammer SM, Eron JJ, Reiss P, Schooley RT, Thompson MA, et al. Antiretroviral treatment of adult hiv infection: 2008 recommendations of the international aids society-USA panel. Jama. 2008; 300 (5): 555-570. Link Retrieved from journal.https://doi:10.1001/jama.300.5.555

46. Zhang X, Sorensen M, Fung MandSchooley R. Synergistic in vitro antiretroviral activity of a humanized monoclonal anti-cd4 antibody (tnx-355) and enfuvirtide (t-20). Antimicrob. Agen. Chemother. 2006; 50: 2231-2233. Link Retrieved from journal

47. Jacobson JM, Israel RJ, Lowy I, Ostrow NA, Vassilatos LS, et al. Treatment of advanced human immunodeficiency virus type 1 disease with the viral entry inhibitor pro 542. Antimicrobial agents and chemotherapy. 2004; 48 (2): 423-429. Link Retrieved from journal.https://doi.10.1128/AAC.48.2.423-429.2004

48. Pugach P, Ketas TJ, Michael EandMoore JP. Neutralizing antibody and anti-retroviral drug sensitivities of hiv-1 isolates resistant to small molecule ccr5 inhibitors. Virology. 2008; 377 (2): 401-407. Link Retrieved from journal.https://doi.org/10.1016/j.virol.2008.04.032

49. Murga JD, Franti M, Pevear DC, Maddon PJandOlson WC. Potent antiviral synergy between monoclonal antibody and small-molecule ccr5 inhibitors of human immunodeficiency virus type 1. Antimicrobial agents and chemotherapy. 2006; 50 (10): 3289-3296. Link Retrieved from journal.https://doi.10.1128/AAC.00699-06

50. Weiner L, Surana RandWang S. Monoclonal antibodies: Versatile platforms for cancer immunotherapy. Nat. Rev. Immunol. 2010; 10: 317-327. Link Retrieved from journal

51. Bhutani DandVaishampayan UN. Monoclonal antibodies in oncology therapeutics: Present and future indications. Expert opinion on biological therapy. 2013; 13 (2): 269-282. Link Retrieved from journal.https://doi.org/10.1517/14712598.2012.758705

52. Mellor JD, Brown MP, Irving HR, Zalcberg JRandDobrovic A. A critical review of the role of fc gamma receptor polymorphisms in the response to monoclonal antibodies in cancer. Journal of hematology \& oncology. 2013; 6 (1): 1. Link Retrieved from journal.https://doi.org/10.1186/1756-8722-6-1

53. Braghiroli MI, Sabbaga JandHoff PM. Bevacizumab: Overview of the literature. Expert review of anticancer therapy. $2012 ; 12$ (5): 567580. Link Retrieved from journal.https://doi.org/10.1586/era.12.13

54. Middleton $\mathrm{K}$, Jones J, Lwin ZandCoward JI. Interleukin-6: An angiogenic target in solid tumours. Critical reviews in oncology/hematology. 2014; 89 (1): 129-139. Link Retrieved from journal.https://doi.org/10.1016/j.critrevonc.2013.08.004

55. Singer J, Fazekas J, Wang W, Weichselbaumer M, Matz M, et al. Generation of a canine anti-egfr (erbb-1) antibody for passive immunotherapy in dog cancer patients. Mol. Cancer Ther. 2014; 13 (1777-1790). Link Retrieved from journal

56. Simpson AandCaballero O. Monoclonal antibodies for the therapy of cancer. BMC Proce. 2014; 8 (S4): 6. Link Retrieved from journal.https://doi.org/10.1186/1753-6561-8-S4-O6

57. Vitale L, Blanset DandLowy I. Prophylaxis and therapy of inhalational anthrax by a novel monoclonal antibody to protective antigen that mimics vaccine-induced immunity. Infect. Immunol. 2006; 74: 5840-5847. Link Retrieved from journal

58. Peterson JW, Comer JE, Noffsinger DM, Wenglikowski A, Walberg KG, et al. Human monoclonal anti-protective antigen antibody completely protects rabbits and is synergistic with ciprofloxacin in protecting mice and guinea pigs against inhalation anthrax. Infection and immunity. 2006; 74 (2): 1016-1024. Link Retrieved from journal.https://doi.10.1128/IAI.74.2.1016-1024.2006

59. Sheoran A, Chapman SandHarvey B. Human antibody against shiga toxin 2 administered to piglets after the onset of diarrhea due to escherichia coli o157: H7 prevents fatal systemic complications. Infect. Immunol. 2005; 73 (4607-4613). Link Retrieved from journal.https://doi.10.1128/IAI.73.8.4607-4613.2005

60. McClain MSandCover TL. Functional analysis of neutralizing antibodies against clostridium perfringens epsilon-toxin. Infection and immunity. 2007; 75 (4): 1785-1793. Link Retrieved from journal.https:/doi.10.1128/IAI.01643-06

61. Kelly-Quintos C, Cavacini LA, Posner MR, Goldmann DandPier GB. Characterization of the opsonic and protective activity against staphylococcus aureus of fully human monoclonal antibodies specific for the bacterial surface polysaccharide poly-nacetylglucosamine. Infection and immunity. 2006; 74 (5): 2742-2750. Link Retrieved from journal.https://doi.10.1128/IAI.74.5.2742$\underline{2750.2006}$

62. Sun Y, Hwang YandNahm M. Avidity, potency, and cross-reactivity of monoclonal antibodies to pneumococcal capsular polysaccharide serotype 6b. Infect. Immunol. 2001; 69: 336-344. Link Retrieved from journal

63. Domanski PJ, Patel PR, Bayer AS, Zhang L, Hall AE, et al. Characterization of a humanized monoclonal antibody recognizing clumping factor a expressed by staphylococcus aureus. Infection and immunity. 2005; 73 (8): 5229-5232. Link Retrieved from journal.https://doi.10.1128/IAI.73.8.5229-5232.2005

64. Hezareh M, Hessell AJ, Jensen RC, van de Winkel JGandParren PW. Effector function activities of a panel of mutants of a broadly neutralizing antibody against human immunodeficiency virus type 1. Journal of virology. 2001; 75 (24): 12161-12168. Link Retrieved from journal.https:doi.10.1128/JVI.75.24.12161-12168.2001

65. Heijtink R, Van Nunen A, van Bergen P, Östberg L, Osterhaus A, et al. Administration of a human monoclonal antibody (tuvirumab) to chronic hepatitis b patients pre-treated with lamivudine: Monitoring of serum tuvirumab in immune complexes. Journal of medical virology. 2001; 64 (4): 427-434. Link Retrieved from journal.https://doi.org/10.1002/jmv.1068

66. Graumann KandPremstaller A. Manufacturing of recombinant therapeutic proteins in microbial systems. Biotechnology Journal: Healthcare Nutrition Technology. 2006; 1 (2): 164-186. Link Retrieved from journal.https://doi.org/10.1002/biot.200500051

67. Beckman RA, Weiner LMandDavis HM. Antibody constructs in cancer therapy: Protein engineering strategies to improve exposure in solid tumors. Cancer. 2007; 109 (2): 170-179. Link Retrieved from journal.https://doi.org/10.1002/cncr.22402 
68. Roopenian DCandAkilesh S. Fcrn: The neonatal fc receptor comes of age. Nature Reviews Immunology. 2007; 7 (9): 715-725. Link Retrieved from journal.https://doi.org/10.1038/nri2155

69. Shinkawa T, Nakamura K, Yamane N, Shoji H, Kanda Y, et al. The absence of fucose but not the presence of galactose or bisecting $\mathrm{n}$ acetylglucosamine of human iggl complex-type oligosaccharides shows the critical role of enhancing antibody-dependent cellular cytotoxicity. J. Biol. Chem. 2003; 278 (5): 3466-3473. Link Retrieved from journal.https://doi.10.1074/jbc.M210665200 